\title{
Angular estimation: An exploratory study
}

\author{
S. GELMINI, M. DALANTIS, and M. S. MAYZNER \\ Loyola University of Chicago, Chicago, Illinois 60626
}

\begin{abstract}
Employing a backward visual masking paradigm, three exploratory studies were carried out to examine the effects of angle size and ISI on angle recognition processing. The results suggest that increasing size probably improves angle recognition performance, but clearly, much additional work remains.
\end{abstract}

As part of an ongoing series of studies (Mayzner, 1975) dealing with pattern recognition, the ability to accurately estimate angle size was investigated. While the following studies were primarily exploratory in nature, it was felt that such research might provide implications for other areas of pattern recognition, such as the recognition of geometric forms or letters.

Stemming in part from previous unpublished work (Mayzner \& Habinek, Note 1), it was felt that the accuracy of angular estimation would improve as angle size increased. Within a backward masking paradigm, subjects were shown a series of angles composed of seven point-light sources and asked to respond in terms of the perceived angle size. It was expected that performance would improve as the angle size increased and as the interval between the angular presentation and the mask presentation increased.

\section{EXPERIMENT 1}

\section{Method}

Subjects. Ten subjects participated in the experiment, all students enrolled in general psychology at Loyola University.

Apparatus. Stimuli were presented on the face of a cathoderay tube (CRT) driven by a PDP-8/e computer, as described by Mayzner (1975). The CRT was in a dimly lighted room adjacent to one containing the computer hardware. Subjects viewed the CRT binocularly through a black viewing tunnel. The tunnel was $60 \mathrm{~cm}$ long and funnel shaped, and it was used to eliminate all external vertical and horizontal cues. Subjects recorded responses on an answer sheet that was provided.

Stimuli. All stimuli were point-light sources arranged according to various spatiotemporal parameters. Test stimuli were angles of $22.5,45.0,67.5,90.0$, and $112.5 \mathrm{deg}$. Each angle was made up of seven points of light, the points being approximately $.833 \mathrm{~cm}$ apart. The vertex of each angle was at the center of the screen, and the angle was positioned in such a way that its bisector was a horizontal line dividing the screen. The mask utilized consisted of three concentric circles of 32 points each and a center point, such that the entire mask consisted of 97 points of light. The mask was constructed in such a way that all points of all angles would be coincident with a point on the mask, with additional points added to the mask so that it was consistent and symmetrical. A fixation point was utilized in the center of the screen at the same location as the vertex of the angles and the center point of the mask.

Procedure. The following procedure was utilized. The

This study was supported by Grant BNS 75-09800 A02 from the National Science Foundation to M. S. Mayzner. subjects sat in the dimly lit room and viewed the stimuli presented on the CRT binocularly through the viewing tunnel. On each trial the fixation point was presented for $1,000 \mathrm{msec}$, followed by a 500-msec delay. One of the five angles was then randomly presented for $10 \mathrm{msec}$; after a variable interstimulus interval (ISI), the mask was presented for $500 \mathrm{msec}$. A total of 150 trials was given to each subject, representing six repetitions of each angle by ISI conditions. The task of the subject was to identify the angle presented.

At the start of each session, the subjects were shown a drawing of each of the angles to be used and were told that the smallest angle would be called $1(22.5 \mathrm{deg})$, the next 2 , and so on, such that the largest (112.5 deg) was 5 . After viewing the angles alone, 10 practice trials were given in exactly the same format as the experimental trials, with the only exception being that the ISIs utilized in the practice trials were of a much greater duration than in the actual trials $(400-100 \mathrm{msec})$. There was a 10 -sec delay between trials that gave the subjects plenty of time to record their responses and to look back through the viewing tunnel in preparation for the next trial. Subjects recorded their own responses; there was no subject-experimenter interaction within sessions after the practice trials, except for a brief resting period between Trials 75 and 76 .

\section{Results}

An analysis of variance with repeated measures yielded significant main effects for the angle size $[F(4,36)=8.986, p<.05]$ and for the ISI times $[F(4,36)=61.113, p<.05]$, and a significant interaction effect $[F(16,144)=2.8137, p<.05]$.

Table 1 shows percent correct responses as a function of ISI. As ISI increased, performance improved, as expected. Table 2 presents the percent correct responses as a function of angle size. There was a gradual improvement in performance as angle size increased, until $90 \mathrm{deg}$ was reached, except with an angle of $112.5 \mathrm{deg}$ when the number of correct responses decreased.

\section{EXPERIMENT 2}

Since the results of Experiment 1 indicated a drop in performance when angle size increased from 90 to $112.5 \mathrm{deg}$, Experiment 2 was carried out to assess whether an angle of $90 \mathrm{deg}$ has some special properties.

\section{Method}

Subjects. Ten undergraduate students enrolled in general psychology at Loyola University were used.

Apparatus. All apparatus utilized was the same as in Experiment 1. 
Table 1

Percent Correct Responses as a Function of ISI

\begin{tabular}{|c|c|c|c|c|c|c|c|c|}
\hline \multirow[b]{2}{*}{ Experiment } & \multicolumn{8}{|c|}{ ISI in Milliseconds } \\
\hline & 5 & 10 & 15 & 20 & 25 & 30 & 40 & 80 \\
\hline 1 & 28.7 & 32.7 & & 43.3 & & & 63.3 & 81.3 \\
\hline 2 & 38.9 & 38.7 & & 50.0 & & & 65.3 & 64.7 \\
\hline 3 & & 60.6 & 66.7 & 61.7 & 58.3 & 71.1 & & \\
\hline
\end{tabular}

Table 2

Percent Correct Responses as a Function of Angle Size

\begin{tabular}{cccccccc}
\hline & \multicolumn{7}{c}{ Angle Size in Degrees } \\
\cline { 2 - 8 } iment & 22.5 & 45.0 & 67.5 & 90.0 & 112.5 & 135.0 & 157.5 \\
\hline 1 & 44.7 & 49.3 & 55.3 & 55.7 & 43.7 & & \\
2 & & & 43.3 & 47.3 & 53.7 & 60.3 & 53.0 \\
3 & & 58.2 & 58.3 & 64.4 & 68.3 & 66.7 & \\
\hline
\end{tabular}

Stimuli. All stimuli were again point-light sources; however, the angles they comprised were $67.5,90.0,112.5,135.0$, and $157.5 \mathrm{deg}$. Again, the angles were made up of seven points of light, with the points approximately $.833 \mathrm{~cm}$ apart. The fixation point and the mask were the same as previously used.

Procedure. The procedure used was the same as in Experiment 1 . Subjects were again shown angles and told to label the smallest $(67.5 \mathrm{deg})$ " 1 " and the largest $(157.5 \mathrm{deg})$ " 5 ." A total of 150 trials were used. There was no experimenter-subject interaction once the trials began, except for the brief resting period at the midpoint of the session.

\section{Results}

An analysis of variance with repeated measures yielded a significant main effect for ISI times $[F(4,36)=$ $57.20, \mathrm{p}<.05]$ and a significant main interaction effect $[F(16,144)=3.2053, p<.05]$. There was no significance found for angle size. Again, see Tables 1 and 2 for the percent correct responses as a function of ISI and angle size, respectively.

\section{EXPERIMENT 3}

At this point, it appeared that performance was improving slightly as angle size increased; however, the effects were not very robust. Therefore, further methodological changes were introduced in Experiment 3.

\section{Method}

Subjects. Twelve subjects enrolled in general psychology at Loyola University participated.

Apparatus. The same apparatus was utilized as in the previous experiments.

Stimuli. All stimuli were again point-light sources, as described previously. All previous angles were utilized, with additional angles of 0 and $180 \mathrm{deg}$, such that there were nine angles in all, differing by $22.5 \mathrm{deg}$. The mask and the fixation point were as previously described.

Procedure. The following procedure was utilized. Subjects sat in the same dimly lit room and viewed the CRT binocularly through the viewing tunnel. Again, for each trial, a fixation point was presented for $1,000 \mathrm{msec}$, followed by a 500 -msec delay, followed by an angle presented for $10 \mathrm{msec}$, a variable ISI, and finally, a mask for $500 \mathrm{msec}$. ISIs utilized were $10,15,20$, and $30 \mathrm{msec}$. Trials were blocked in seven sets of 15 trials each, such that there were a total of 105 trials.
At the start of each session, subjects were shown three angles on the CRT, $0,22.5$, and $45.0 \mathrm{deg}$, and told that the smallest would be called 1 , and middle size 2 , and the largest 3 . The format of the experiment was then explained, and the subject was instructed to write the appropriate number of his answer sheet for each trial. Fifteen trials were than given with the angle size (0-45 deg) and the ISI randomly presented, with three repetitions of each condition. Upon completion of this block of trials, the subject was shown three new angles on the CRT, $22.5,45.0$, and $67.5 \mathrm{deg}$, and instructed to again respond by recording a 1,2 , or 3 for each trial. Five more blocks of trials were given such that there were blocks with angles of 45.0, 67.5 , and $90.0 \mathrm{deg} ; 67.5,90.0$, and $112.5 \mathrm{deg} ; 90.0,112.5$, and $135.0 \mathrm{deg} ; 112.5,135.0$, and $157.5 \mathrm{deg}$; and 135.0, 157.5, and $180.0 \mathrm{deg}$. All responses were recorded by the subjects. At the end of the first block there was a chance for the subjects to ask questions concerning the format of the experiment, and, following that, there was no experimenter-subject interaction.

\section{Results}

Only responses corresponding to 45 - to $135-\mathrm{deg}$ angles were scored, as only these angles were presented an equal number of times. An analysis of variance with repeated measures yielded a significant main effect for ISI time $[F(4,44)=21.372, p<.05]$. There was no significant interaction effect found, nor was there a significant main effect found for angle size. Tables 1 and 2 present the percent correct responses as a function of ISI and angle size.

\section{DISCUSSION}

It was expected that performance would improve both as ISI increased, and as the size of the angle presented increased. Performance as a function of angle size improved significantly only in the first study. Although nonsignificant in the following two studies, it should be noted that there was a slight trend evident, in that performance did improve slightly with the interesting exception of the largest angle presented in all cases. This is especially odd in the third study, as the largest angle scored was not the largest angle tested; there were actually two larger angles presented within the experimental trials. It was partially this phenomena in Experiments 1 and 2 that provided the impetus for Experiment 3, in which the end angles (largest and smallest) were not scored.

This short series of studies was intended to be exploratory in nature. It was felt that some insight into the recognition of angles might provide for a better understanding of patterns. There did seem to be limited evidence that larger angles were easier to correctly recognize than others. It was also interesting to note that the 90-deg angle was not significantly easier to recognize than the others, as might have been expected, due to its familiarity. Further, there seems to be a differential effect with respect to angle size, yet this effect is slight, clearly suggesting a need for additional study. Again, it is interesting to note that there was always a performance drop with the fifth angle tested, suggesting that the problem of relative judgments 
may affect angle recognition processes in this type of paradigm.

\section{REFERENCE NOTE}

1. Mayzner, M. S., \& Habinek, J. K. Studies of angular recognition processes. Unpublished study, 1976.

\section{REFERENCE}

MAYZnER, M. S. Studies of visual information processing in man. In R. L. Solso (Ed.), Information processing and cognition: The Loyola symposium. Hillsdale, N.J: Erlbaum, 1975.

(Received for publication July 10, 1979.) 